\title{
Zusammenleben. Deutschtum und Judentum aus jüdischer Sicht (1912/1915)
}

Vivre ensemble. Germanité et judéité dans une perspective juive (1912/1915)

Living together. Germanness and Jewishness from a Jewish Perspective

(1912/1915)

\section{Sonia Goldblum}

\section{(2) OpenEdition}

\section{Journals}

Édition électronique

URL : https://journals.openedition.org/ceg/6938

DOI : $10.4000 /$ ceg. 6938

ISSN : 2605-8359

Éditeur

Presses Universitaires de Provence

Édition imprimée

Date de publication : 25 septembre 2019

Pagination : $39-52$

ISBN : 979-10-320-0234-6

ISSN : 0751-4239

Référence électronique

Sonia Goldblum, „Zusammenleben. Deutschtum und Judentum aus jüdischer Sicht (1912/1915)",

Cahiers d'Études Germaniques [Online], 77 | 2019, Online erschienen am: 25 März 2021, abgerufen am

15 Juni 2021. URL: http://journals.openedition.org/ceg/6938 ; DOI: https://doi.org/10.4000/ceg.6938 


\section{Zusammenleben. Deutschtum und Judentum aus jüdischer Sicht (1912/1915)}

Sonia GOLDBLUM

Université de Haute-Alsace - Mulhouse / FRIAS Universität Freiburg / ILLE (4363)

\section{Deutschtum und Judentum - zur Einführung}

Die folgenden Ausführungen gehen der Frage nach, wie die deutschen Juden in den 1910er Jahren und in der Zwischenkriegszeit sich das Zusammenleben mit nichtjüdischen Deutschen in Deutschland vorstellten und wie sie darüber reflektierten. Der gewählte Titel übernimmt die Formulierung der Akteure dieser Debatten, um ihren problematischen Charakter zu unterstreichen.

Es ist sicherlich möglich und sinnvoll, über die Bedingungen eines Zusammenlebens von nichtjüdischen und jüdischen Deutschen nachzudenken — und tatsächlich wurde diese Reflexion von einzelnen Autoren auch geführt. Im Fokus der abstrakten Kategorien „Deutschtum" und „Judentum" stehen aber nicht so sehr die Menschen: Diese Begriffe, die im Diskurs über das Zusammenleben von Juden und Nichtjuden und über die Aufnahmebereitschaft der deutschen Gesellschaft gegenüber den Juden vielfach benutzt wurden, bergen die Gefahr einer Essentialisierung. Dennoch wurden sie damals selten in Frage gestellt.

Anhand ausgewählter Texte, die hier zusammen mit einigen der Reaktionen, die sie hervorriefen, präsentiert werden, lässt sich die Grundsatzfrage nach der Möglichkeit eines Lebens in der Diaspora diskutieren. Pauschal lassen sich dabei drei Positionen erkennen, die aber natürlich nicht als komplett einheitlich anzusehen sind. Auf der einen Seite vertraten die meisten Zionisten die Idee, dass das Leben in der Diaspora nicht mehr wünschenswert oder nicht mehr zeitgemäß sei. Am anderen Ende des Spektrums findet man eine Reihe von Persönlichkeiten, die dem nationalliberalen Lager zuzuordnen sind und die Meinung äußerten, dass der Weg zur "Assimilation", d.h. das Aufgehen der Juden in der deutschen Gesellschaft noch abgeschlossen werden solle, damit die Juden in Deutschland vollkommen akzeptiert würden. Politisch war letztere Tendenz hauptsächlich durch den Centralverein deutscher Staatsbürger jüdischen Glaubens (CV) 
vertreten. ${ }^{1}$ Zwischen diesen beiden Polen befanden sich Personen, die weder bereit waren, das Leben in Deutschland aufzugeben, noch für eine radikale Assimilation plädierten. Waren schon die beiden ersten Gruppen sehr heterogen, so war die dritte noch vielfältiger.

Im vorliegenden Aufsatz sollen vor allem zwei prominente Debatten beleuchtet werden. Sie ermöglichen eine Herausarbeitung der verschiedenen Positionen und zeigen exemplarisch, von welcher Art die Debatten im Deutschland der 1910er Jahre waren, als die Bewegung einsetzte, die man gewöhnlich mit Martin Buber als „Jüdische Renaissance" bezeichnet. ${ }^{2}$ Diese Bewegung setzte sich dann zwischen dem Ersten Weltkrieg und der nationalsozialistischen "Machtübernahme" im Jahre 1933 fort; unter ihrer Ägide wurde eine Reihe von Projekten durchgeführt, die das Ziel hatten, das jüdische Selbstverständnis zu stärken. Man denke zum Beispiel an die "Lehrhausbewegung" oder an andere Institutionen zur Pflege und wissenschaftlichen Untersuchung jüdischen Wissens. ${ }^{3}$

\section{Die Debatten in ihrem Kontext}

Der Bankier und Nationalliberale Ludwig Bamberger (1823-1899) war der erste Publizist, der eine Schrift mit demTitel,Deutschtum und Judentum“ veröffentlichte. Bambergers Schrift ist im Kontext des Berliner "Antisemitismusstreits" (1879-1881) ${ }^{4}$ zu verstehen: Sie gehört nämlich zu den zahlreichen Reaktionen, die Heinrich von Treitschkes Artikel „Unsere Aussichten“ aus dem Jahr 1879 hervorrief. Der dritte Teil der Abhandlung Treitschkes befasst sich mit der so genannten Judenfrage und lässt sich mit dem bekannten Ausruf zusammenfassen: „Die Juden sind unser Unglück". ${ }^{5}$ Treitschke spricht den Juden die Fähigkeit ab, in der deutschen Nation aufzugehen, denn „[e]ine Kluft zwischen abendländischem und semitischem Wesen ha[be] von jeher bestanden". ${ }^{6}$

1. Avraham Barkai, „,Wehr dich!“ Der Centralverein deutscher Staatsbürger jüdischen Glaubens (C.V.) 1893-1938, München, Beck, 2002.

2. Martin Buber, „Jüdische Renaissance", Ost und West 1, 1901, S. 7-10.

3. Das Paradebeispiel dafür ist das von Franz Rosenzweig 1920 gegründete jüdische Lehrhaus in Frankfurt. Die jüdische Volkshochschule und die Akademie für die Wissenschaft des Judentums in Berlin könnten in diesem Zusammenhang auch genannt werden. Vgl. Sonia Goldblum, „Jüdisches Forschen oder jüdisches Lernen. Zwei jüdische Wissenseinrichtungen in Berlin in den 1920er Jahren", in Laurence Guillon, Heidi Knörzer (Hrsg.), Berlin und die Juden. Geschichte einer Wahlverwandtschaft?, Berlin, Neofelis, S. 89-108.

4. Vgl. Stanley Zucker, "Ludwig Bamberger and the Rise of Antisemitism in Germany, 1848-1893", Central European History 3 (4), 1970, S. 332-352; Uffa Jensen, Gebildete Doppelgänger: bürgerliche Juden und Protestanten im 19. Jahrhundert, Göttingen, Vandenhoeck \& Ruprecht, 2005, S. 226229.

5. Die komplette Debatte mit all den dazu gehörigen Texten und Kommentaren wurde in einer zweibändigen Quellenausgabe rekonstruiert. Karsten Krieger (Hrsg.), Der „Berliner Antisemitismusstreit" 1879-1881, München, Saur, 2003. Vgl. Ludwig Bamberger, ,Deutschtum und Judentum", in ibid., S. 217-243; Heinrich von Treitschke, „Unsere Aussichten", in ibid., S. 6-16, hier S. 14.

6. Ibid., S. 15. 
In den späteren Debatten, die in den folgenden Ausführungen präsentiert werden, ging es aber weniger darum, den Antisemitismus zu analysieren oder zu bekämpfen, als vielmehr darum, die Position der Juden in Deutschland und ihr Verhältnis zu den nichtjüdischen Deutschen zu bestimmen. Der Antisemitismus blieb zwar im Diskurs präsent, aber er spielte nicht mehr eine so herausragende Rolle wie im „Antisemitismusstreit". Dies hat auch mit dem Kontext zu tun, in dem die Texte entstanden und die Debatten geführt wurden.

Moritz Goldsteins Text „Deutsch-jüdischer Parnass” erschien im März 1912 in der konservativen Zeitschrift Der Kunstwart. ${ }^{7}$ Als Herausgeber der Goldenen Klassikerbibliothek war Goldstein (1880-1977) im damaligen literarischen Feld kein Unbekannter. "Deutsch-jüdischer Parnass" war auch nicht sein erster Beitrag zum Thema des jüdischen Lebens in Deutschland. 1906 hatte er bereits einen Essay mit dem Titel "Geistige Organisation des Judentums" in Ost und West veröffentlicht, der aber bei Weitem nicht den Widerhall des Kunstwart-Aufsatzes gefunden hatte. ${ }^{9}$ Das Erscheinungsdatum dieses Essays ist alles andere als zufällig. Der März 1912 war nämlich der hundertste Jahrestag des „Preußischen Judenedikts" von 1812, das für die deutschen Juden den ersten Schritt auf dem Weg zur Gleichberechtigung darstellte: „Die in Unsern Staaten jetzt wohnhaften, mit General-Privilegien, Naturalisationspatenten, Schutzbriefen und Konzessionen versehenen Juden und deren Familien sind für Einländer und preußische Staatsbürger zu achten“. ${ }^{10}$ Goldsteins Text ist also eine Art Bilanz der Situation der Juden im literarischen Feld hundert Jahre nach dem Anfang der Emanzipationsbewegung, obwohl er diesen Jahrestag selbst nie erwähnt. Da um diese Zeit einige Texte in der jüdischen Presse erschienen, die explizit Bezug auf das Judenedikt nahmen, kann man sich nur schwer vorstellen, dass Goldstein diesen Umstand nicht mitbedacht hatte. ${ }^{11}$

Drei Jahre später, im Juli 1915, erschien ein Text des neukantianischen Philosophen Hermann Cohen mit dem Titel Deutschtum und Judentum. Mit grundlegenden Betrachtungen über Staat und Internationalismus ${ }^{12}$. Der Kontext des Krieges erklärt den dezidierten Patriotismus, den Cohen in diesem Text ständig bemüht. Der „Burgfrieden“, den Wilhelm II. am 4. August 1914 verkündet hatte, hatte bei vielen deutschen Juden eine große Hoffnung hervorgerufen. Sie sahen im Krieg die Möglichkeit, ihre Loyalität gegenüber Deutschland unter Beweis zu stellen und damit dem Antisemitismus ein Ende zu setzen. ${ }^{13}$ Cohens

7. Moritz Goldstein, „Deutsch-jüdischer Parnass“, Der Kunstwart 25 (11), 1912, S. 281-294.

8. Andreas Kilcher, "'Goldstein Moritz", in Ders. (Hrsg.), Deutsch-jüdische Literatur. 120 Porträts, Berlin, Metzler, 2006, S. 72-75.

9. Moritz Goldstein, „Geistige Organisation des Judentum”, Ost und West 8-9, 1906, S. 513-526.

10. [https://babel.hathitrust.org/cgi/pt?id=uc1.b3094006;view=1up;seq=427], Stand: 14. Januar 2019.

11. Vgl. Heinrich Margulies, „Das Jahrhundert der deutsch-jüdischen Kulturmischung”, Jüdische Rundschau 12, 1912, S. 100.

12. Hermann Cohen, Deutschtum und Judentum, mit grundlegenden Betrachtungen über Staat und Internationalismus, Gießen, A. Töpelmann, 1915.

13. Michael Meyer, Michael Brenner, Deutsch-jüdische Geschichte in der Neuzeit, Bd. 3, 1871-1918, hrsg. v. Steven Lowenstein, Paul Mendes-Flohr, Peter Pulzer et al., München, Beck, 2000, S. 362. 
Text wurde ein Jahr vor der so genannten Judenzählung von 1916 publiziert, die all diese Hoffnungen zunichte machte. ${ }^{14}$

Die 1910er Jahre stellen also eine Phase des Übergangs dar, bevor die Selbstbehauptung der deutschen Juden in der Weimarer Republik sich auf alle Bereiche der jüdischen Kultur und des jüdischen Alltagslebens ausdehnte. ${ }^{15}$ An den jeweiligen Texten soll nun exemplarisch gezeigt werden, was ihre Autoren unter, ,Volk' und ,Nation', sowie ,Emanzipation' und ,Assimilation' verstanden. ${ }^{16}$

\title{
Die Kunstwartdebatte 1912 - Die Juden als Öffentlichkeit
}

\author{
Im Märzheft 1912 der Zeitschrift Der Kunstwart wird Moritz Goldsteins Essay eine \\ kurze Bemerkung des Herausgebers Ferdinand Avenarius vorangestellt:
}

\begin{abstract}
Unter dieser Überschrift sendet uns ein gebildeter Jude Ausführungen, die schon um ihrer ganz ungewöhnlichen Offenherzigkeit willen bei Juden wie Nichtjuden Aufsehen erregen dürften. Es versteht sich von selbst, dass wir sie nicht als unsre eignen Meinungen wiedergeben, und dass dieser Beleuchtung von einer Seite die Beleuchtung von andern her folgen muss. Aber Goldsteins Aufsatz scheint uns ganz ungewöhnlich geeignet als Ausgangspunkt [Hervorhebung im Original, SG] einer Erörterung. ${ }^{17}$
\end{abstract}

Damit distanziert sich Avenarius von Goldsteins Stellungnahmen: Sie entsprächen weder seiner Meinung noch der Ausrichtung der Zeitschrift. Gleichzeitig sieht er sie von vornherein als Ausgangspunkt einer Debatte, die in den folgenden Monaten im Rahmen der Zeitschrift auch tatsächlich stattfand. Der Kunstwart publizierte nämlich im April- bzw. im Augustheft unter der Überschrift "Sprechsaal“ einige der Zuschriften, die die Redaktion nach der Publikation von „Deutsch-jüdischer Parnass" erhalten hatte. ${ }^{18}$

Goldsteins Argumentation bezieht sich zunächst auf die deutsche Literatur. Ein Satz insbesondere erregte viel Aufsehen und wurde immer wieder zitiert und kommentiert: „Wir Juden verwalten den geistigen Besitz eines Volkes, das uns die Berechtigung und die Fähigkeit dazu abspricht" ${ }^{\prime 19}$, so Goldsteins Diagnose über die Rolle der Juden im deutschen Kulturleben. Damit ist sowohl die herausragende Rolle einiger deutscher Juden im kulturellen und intellektuellen Bereich angesprochen, als auch die Verachtung, ja die Feindschaft, denen sie

14. Der offizielle Name dieser Maßnahme lautet: „Nachweisung der beim Heere befindlichen wehrpflichtigen Juden".

15. Vgl. Michael Brenner, Jüdische Kultur in der Weimarer Republik, aus dem Englischen v. Holger Fliessbach, München, Beck, 2000, S. 21-46.

16. Vgl. auch Meyer, Brenner, Deutsch-jüdische Geschichte in der Neuzeit, Bd. 3, S. 335-345.

17. Goldstein, „Deutsch-jüdischer Parnass“, S. 281. Avenarius' Vorspann bildet eigentlich den ersten Absatz von Goldsteins Essay. Er trägt keine Überschrift, und der Name des Autors wird auch nicht angegeben. Der Inhalt lässt aber eindeutig schließen, dass es sich um den Chefredakteur der Zeitschrift handeln muss.

18. "Sprechsaal. Unter sachlicher Verantwortung der Einsender. Aussprache zur Judenfrage”, Der Kunstwart 25 (13), 1912, S. 6-15. „Sprechsaal. Unter sachlicher Verantwortung der Einsender. Aussprache zur Judenfrage“, Der Kunstwart 25 (22), 1912, S. 236-261.

19. Goldstein, „Deutsch-jüdischer Parnass”, S. 283. 
immer wieder ausgesetzt waren. Eine paradoxe Situation wird also skizziert, in der die Juden gleichzeitig präsent seien und doch nicht willkommen. Allerdings kann man mit Steven Loewenstein zu bedenken geben, dass der "beträchtliche Anteil von Juden an der deutschen Kultur" nicht als "das Aufkommen eines fremden Einflusses“ gedeutet werden darf, sondern als Zeichen dafür, „wie ,germanisiert' die deutschsprachigen Juden geworden waren". 20

Außerdem sollte nicht vergessen werden, dass es in Goldsteins Text nicht nur um eine Diagnose, sondern auch um ein Programm geht, das im Sinne der Buberschen „Jüdischen Renaissance“21 für eine Veränderung des Selbstverständnisses der Juden in Deutschland plädiert: Problematischer als die mangelnde Anerkennung von deutscher Seite ist für Goldstein das mangelnde Selbstbewusstsein der Juden und das Fehlen einer „jüdischen Öffentlichkeit“.22 Diese Situation, die er feststellt, zwinge ihn „vor aller Welt zu sagen, was [er] nur vor Juden sagen sollte".23 Und darin besteht der zweite Teil der Diagnose: „Es ist in Deutschland, überhaupt in Westeuropa, nicht möglich, zur Gesamtheit der Juden als Juden zu sprechen, soviel wir auch über uns sprechen lassen müssen". ${ }^{24}$ Dies wird im ersten Teil des Textes ausgeführt. Goldstein kommt auf den Antisemitismus im literarischen Feld zu sprechen, aber im Gegensatz zu Bamberger, der „Bildung und Humanität” als Waffen gegen den Antisemitismus ansah, geht es ihm nicht vordergründig darum, die Gründe des Antisemitismus aufzudecken und Lösungen zu finden. Stattdessen schlägt er folgendes Programm vor:

[D]ie nationale Eigenart muß sich so steigern, verinnerlichen, veredeln lassen, daß sie zum Vorzug, zur Tugend wird, daß eine besondere Kraft und alle Leistungen daraus hervorquellen. Denn wenn die Juden nicht als Juden etwas wert sind, so sind sie überhaupt nichts wert.

Der Gebrauch des Adjektivs "national“, um das jüdische Selbstverständnis zu charakterisieren, signalisiert Goldsteins Zustimmung zur zionistischen Idee. Es geht ihm nicht um religiöse bzw. konfessionelle Zugehörigkeit, wie sie etwa von den Vertretern der Emanzipationsbewegung aufgefasst wurde, sondern um eine Schicksalsgemeinschaft, die er im Sinne eines Ausrufs zum Nationalstolz zum Ausdruck bringt. Er unterstützt also das zionistische Projekt und befürwortet den "Sprung in die neuhebräische Literatur". ${ }^{25}$ Dies sei aber, so seine Ansicht, für seine Generation - 1912 ist er 32 Jahre alt - keine Lösung. Er schlägt also einen anderen Weg vor, der ein „[S]ich abfinden“"26 abmildern, ja mit Sinn füllen kann:

20. Steven M. Lowenstein, „Der jüdische Anteil an der deutschen Kultur”, in Meyer, Brenner, Deutschjüdische Geschichte in der Neuzeit, Bd. 3, S. 302-332, hier S. 302. Lowenstein betont zunächst, wie schwierig es ist, diesen Anteil genau zu bemessen, bevor er selbst ein möglichst genaues Panorama davon liefert.

21. Till Schicketanz, "Im Schatten des "Parnaß'. Moritz Goldstein und die Jüdische Renaissance", Aschkenas 18-19 (2), 2008-2009, S. 249-267.

22. Goldstein, „Deutsch-jüdischer Parnass", S. 282.

23. Ibid.

24. Ibid.

25. Ibid., S. 290.

26. Ibid. 
Unser Verhältnis zu Deutschland ist das einer unglücklichen Liebe: wir wollen endlich männlich genug sein, uns die Geliebte, statt ihr endlos kläglich nachzuschmachten, mit kräftigem Entschlusse aus dem Herzen zu reißen - und bleibe auch ein Stück Herz hängen. ${ }^{27}$

Die Lösung, die Goldstein als „Linderungsmittel“ bezeichnet, ist einfach: „sich laut und rücksichtslos, ich möchte beinahe sagen, schamlos als Juden bekennen“.28 Es geht ihm darum, dass die Juden offen zur eigenen Identität stehen und es sich nicht mehr gefallen lassen, dass das Wort "Jude" von Nichtjuden als Schimpfwort benutzt wird. Hier haben wir es mit einem der wichtigsten Merkmale der jüdischen Renaissance zu tun. Wichtig ist nicht nur die Kritik am Emanzipationsprozess, der als Prozess des Selbstverlusts, oder gar der Selbstverleugnung angeprangert wird $^{29}$, sondern vor allem die Bemühung um eine positive Definition des Judentums: Diese soll keine Fremdbezeichnung sein, die im Zusammenhang mit der "Judenfrage" oder mit der Reaktion auf den Antisemitismus steht, sondern eine genuine Selbstbehauptung.

Avenarius sah in diesem Text den Ausgangspunkt einer Debatte, und tatsächlich erhielt er in Folge der Publikation um die neunzig Zuschriften. ${ }^{30}$ Die Debatte wurde sowohl in Der Kunstwart als auch - wenn auch in geringerem $\mathrm{Maße}$ - in der jüdischen Presse geführt. ${ }^{31}$ Der Kunstwart publizierte im April- bzw. im Augustheft 1912 einige Reaktionen unter der Rubrik „Sprechsaal“. Die jüdische Presse war viel weniger geneigt, sich in diese Debatte einzumischen. Tatsächlich weigerte sich die Allgemeine Zeitung des Judentums, dem Thema eigene Artikel zu widmen. Diese verhaltene Position erklärt sich wahrscheinlich dadurch, dass die Zeitung als unparteiisch galt und deshalb nicht auf eine Stellungnahme festgelegt werden wollte. Dennoch publizierte der Herausgeber Ludwig Geiger im November 1912 einen Leitartikel, in dem er Goldsteins Position ausdrücklich ablehnte und an ihm kein gutes Haar ließ. ${ }^{32}$

27. Ibid., S. 292

28. Ibid.

29. In den 1960er Jahren wurde die Thematik der Selbstverleugnung, ja der "Selbstaufgabe" bei Autoren wie Gershom Scholem prominent, die entschieden gegen die Existenz einer deutschjüdischen Symbiose vor 1933 argumentierten. Vgl. Gershom Scholem, „Wider den Mythos vom deutsch-jüdischen ,Gesprächs'", in ders. Judaica 2, Frankfurt a. M., Suhrkamp, 1970, S. 7-11, hier S. 9.

30. Kilcher, „,Goldstein“, S. 73. Zu dieser Debatte, vgl. auch Irmtraud Ubbens, „Moritz Goldstein:, ,... die anderen fühlen uns ganz undeutsch'. 100 Jahre Deutsch-jüdischer Parnaß. Eine Kulturdebatte in der jüdischen Presse (1912)“, Medaon 12, 2013, http://www.medaon.de/pdf/MEDAON_12_ Ubbens.pdf [Stand: 10. Februar 2019].

31. Alle für die Kunstwart-Debatte relevanten Texte wurden in folgendem Band versammelt und herausgegeben: Julius H. Schoeps (Hrsg.), Deutsch-jüdischer Parnaß. Rekonstruktion einer Debatte (Menora. Jahrbuch für deutsch-jüdische Geschichte 13), Berlin/ Wien, Philo, 2002. Die verschiedenen Ebenen, auf denen die Debatte sich abspielte, werden in folgendem Aufsatz rekonstruiert: Manfred Voigts, „Die ,Kunstwart-Debatte' - Kontroversen um Assimilation und Kulturzionismus", in Horch, Handbuch der deutsch-jüdischen Literatur, Berlin, De Gruyter, S. 122134.

32. Vgl. Ludwig Geiger, „Der Kunstwart und die Judenfrage“, Allgemeine Zeitung des Judentums Nr. 46, 15. November 1912. 
Ernst Lissauer, einst vom Literaturwissenschaftler Walter A. Berendsohn als "deutscheste[r] aller jüdischen Dichter" bezeichnet, publizierte im Aprilheft von Der Kunstwart eine Besprechung, in der er vor allem die Assimilation verteidigte. ${ }^{33} \mathrm{Er}$ warf Goldstein eine oberflächliche Herangehensweise vor, die von seinem Mangel an historischem und soziologischem Bewusstsein herrühre. ${ }^{34}$ Lissauer fasste ein Volk als "geschichtliche[s] Resultat des Zusammenlebens [der] Menschen“ auf. ${ }^{35}$ Seiner Meinung nach entsprächen die Juden nicht dieser Definition, da sie in der Diaspora zerstreut lebten. Dies gab dem Autor die Gelegenheit, seine antisemitisch anmutenden Ansichten gegenüber den Ostjuden und dem jiddischen „Jargon" - wie er ihn nennt -, zum Besten zu geben. ${ }^{36}$ Lissauer zufolge bildeten auch die deutschen Juden kein Volk, obwohl sie das Kriterium des Zusammenlebens erfüllen würden. Wer die Juden als ein Volk betrachte, so Lissauer, nutze dieselben Argumente wie die Antisemiten, die "das Dogma der Unveränderlichkeit der semitischen Rasse" vertreten würden. ${ }^{37}$ Der Artikel schließt mit folgender Alternative ab: „Nur zweierlei ist möglich: entweder: auswandern; oder: deutsch werden". ${ }^{38}$ Damit wird der Zionismus und die für ihn charakteristische Form des Nationalismus als ein anders gedrehter Antisemitismus abgetan und schließlich das assimilatorische Projekt auf eine Weise verfochten, die in ihrer Konsequenz polemische Züge annimmt.

Die zweite Erwiderung, die nun präsentiert wird, stammt aus dem entgegengesetzten Lager. Sie wurde von Ludwig Strauß verfasst, einem Dichter, Vertreter des Kulturzionismus und Martin Bubers späterem Schwiegersohn. Sie erschien unter dem Pseudonym Franz Quentin im Augustheft 1912 des Kunstwart. Da Strauß' Reaktion sich sowohl gegen Goldstein als auch gegen Lissauer richtet, ist sie in unserem Zusammenhang von besonderem Interesse. ${ }^{39}$ Im Gegensatz zu Lissauer besteht Strauß darauf, dass die Zugehörigkeit zu einem

33. Walter A. Berendsohn, Die humanistische Front. Einführung in die deutsche Emigrantenliteratur, Teil 1, Zürich, Europa Verlag, 1946, S. 54f. Zu Ernst Lissauer, vgl. auch Christian Wiese, Lissauer, Ernst, in Andreas B. Kilcher (Hrsg.), Metzler Lexikon der deutsch-jüdischen Literatur. Jüdische Autorinnen und Autoren deutscher Sprache von der Aufklärung bis zur Gegenwart, Stuttgart/ Weimar, Metzler, 2012, S. 342-344.

34. Zu der Art und Weise, wie die jüdische Geschichtswissenschaft die Emanzipations- bzw. Assimilationsbewegung rechtfertigte, vgl. Andreas Gotzmann, "Geschichte in Abkehr von der Vergangenheit. Zur Problematik historischer Identität im deutschen Judentum der Emanzipationszeit", Aschkenas 9 (2), 1999, S. 327-351, hier S. 343.

35. Ernst Lissauer, ohne Titel, Der Kunstwart 25 (13), 1912, S. 6-12, hier S. 6. Diese Definition stammt laut Lissauer vom „Junker von der Marwitz“. Es gibt Gründe anzunehmen, dass es sich dabei um Friedrich August Ludwig von der Marwitz handelt, der 1811 zusammen mit Friedrich Ludwig Karl Graf Finck von Finckenstein die so genannte Lebuser Denkschrift verfasste. Sie richtete sich gegen die von Hardenberg geführte Reform der Ständegesellschaft, enthielt aber auch deutlich antisemitische Passagen. Vgl. Marco Puschner, Antisemitismus im Kontext der Politischen Romantik. Konstruktionen des "Deutschen" und des „Jüdischen" bei Arnim, Brentano und Saul Ascher, Tübingen, Max Niemeyer, 2008, S. 200.

36. Lissauer, ohne Titel, S. 7.

37. Ibid., S. 9.

38. Ibid., S. 12.

39. Ludwig Strauß, ohne Titel, Der Kunstwart 25 (25), 1912, S. 238-244. Dazu vgl. Itta Shedletzky, „Fremdes und Eigenes. Zur Position von Ludwig Strauß in den Kontroversen um Assimilation und 
Volk abstammungsbedingt sei, und plädiert aus diesem Grund für eine jüdische Renaissance, deren Avantgarde die Zionisten bilden sollen. Seine Ausführungen orientieren sich stark an Martin Bubers „Drei Reden über das Judentum”, die er ausgiebig zitiert. ${ }^{40}$ Für ihn "erzeugt" der Kontakt der Juden mit dem "Wirtsvolk" einen neuen Typus, der sowohl von demjenigen des Deutschen als auch von dem des "Volljuden“ abweiche. ${ }^{41}$ Die Wortwahl ist interessant, und man würde gerne fragen, was Strauß sich unter einem "Volljuden“ vorstellt: Meint er damit die Ostjuden, die der Tradition, so ein damals weit verbreiteter Glaube, näher geblieben seien als die deutschen Juden? Meint er eher die Juden vor dem Anfang der Emanzipationsbewegung, oder handelt es sich um ein gedankliches Konstrukt, das der abstrakten Idee eines Wesens des Judentums nahekommen würde? Strauß scheint hier mit einer Fiktion der Authentizität und Ursprünglichkeit zu operieren, die er nicht näher erläutert. Aus diesen Ausführungen schließt er auf die Unmöglichkeit eines Aufgehens der Juden in der Gesellschaft des jeweiligen Landes und auf die Notwendigkeit einer Emigration nach Palästina, die er 1935 tatsächlich vollzog. ${ }^{42}$

Bezüglich der Kunstwart-Debatte kann man festhalten, dass Goldstein mit seiner Diagnose und seiner doppelten Lösung es niemandem recht machen konnte. Die Anhänger eines strengen Assimilationsprozesses konnten weder seine pessimistische Diagnose noch seine beiden Lösungen der Selbstbehauptung bzw. des Sprungs in die hebräische Sprache akzeptieren. Den Zionisten war sein Lippenbekenntnis zu wenig dezidiert. Nichtsdestoweniger trat Goldstein in diesem Text für eine jüdische Selbstbehauptung in der Diaspora ein, die als Vorwegnahme von Rosenzweigs Begriff der "Dissimilation" gelten kann. ${ }^{43}$

\section{Deutschtum und Judentum. Volk als Volksgeist}

Im Folgenden soll kurz auf die eigentümliche Rhetorik Hermann Cohens in seinem Werk Deutschtum und Judentum (1915) eingegangen werden, so wie auf zwei verschiedene Reaktionen, die von diesem Buch hervorgerufen wurden. Cohens Text fängt folgendermaßen an:

Die Vergleichung von Volksgeistern bringt die Gefahr mit sich, daß das Eigentümliche eines jeden derselben bei der Vergleichung nicht in voller Genauigkeit und Bestimmtheit zur

Judentum in den Jahren 1912-1914“, in Hans Otto Horch, Ludwig Strauß 1892-1992. Beiträge zu seinem Leben und Werk, Tübingen, Niemeyer, 1995, S. 174-183.

40. Martin Buber, „Drei Reden über das Judentum“, in Ders. Frühe jüdische Schriften, hrsg. v. Barbara Schäfer, Gütersloh, Gütersloher Verlagshaus, 2007, S. 219-256.

41. Ludwig Strauß, ohne Titel, S. 241.

42. Strauß gehört zu den wenigen Literaten, denen der von Goldstein gewünschte „Sprung in die neuhebräische Literatur" tatsächlich gelungen ist. Vgl. Sonia Goldblum, „Von Jerusalem nach Weimar. Rückblick auf die deutsch-jüdischen Beziehungen in der Zwischenkriegszeit", in Katja Schubert, Laurence Guillon (Hrsg.), Deutschland und Israel/ Palästina von 1945 bis heute, Würzburg, Königshausen \& Neumann 2014, S. 35-50, hier S. 48.

43. Franz Rosenzweig, Briefe und Tagebücher, hrsg. v. Rachel Rosenzweig, Dordrecht, Nijhoff, 1979, S. 770. Dazu vgl. auch Stéphane Mosès, L’Ange de I'Histoire, Paris, Gallimard, 2006, S. 49-82. 
Ermittlung kommen könnte. Diese Gefahr aber wird geringer, wenn die zu vergleichenden Volksgeister mit einem dritten Volksgeiste eine innerliche Verbindung eingegangen sind, und zwar nicht nur einmal, sondern wiederholentlich. ${ }^{44}$

Dieser Auszug ist beispielhaft für Cohens hoch spekulative Ausdrucksweise. Er sieht sein Unterfangen als ein philosophisches an und bedient sich einer Sprache, die ihn in Hegels idealistische Tradition stellt. Seine Art der Beweisführung würde man heute wohl als essentialistisch bezeichnen. Es geht ihm nicht um eine Beschreibung der konkreten, historischen Situation der Juden in Deutschland, sondern um eine Wesensbestimmung von "Deutschtum" und "Judentum". Der Begriff "Volksgeist", den Cohen eingangs verwendet, ist in diesem Kontext zu verstehen. ${ }^{45}$ Eine andere Quelle des Begriffs ist sicherlich auch die Völkerpsychologie, die Hermann Cohen maßgeblich beeinflusste. ${ }^{46} \mathrm{Er}$ vergleicht streng genommen nicht die Völker, was die Frage nach ihrer genauen Definition aufwerfen würde: Soll das Volk als Gruppe von einzelnen Individuen oder als Ganzheit, als einheitliche Gemeinschaft aufgefasst werden? Dadurch, dass Cohen auf den "Volksgeist" abhebt, stellt er sich auf eine abstrakte Metaebene, die die Relevanzsolcher Fragen aushebelt. Allerdings scheinter sich der Gefahr bewusstzu sein, die seinen Äußerungen innewohnt, und versucht ihr durch das Heranziehen unterschiedlicher Vergleichsgrößen - allen voran Christentum und Griechentum - zu entgehen. Dazu schreibt er: „Diese Gefahr aber wird geringer, wenn die zu vergleichenden Volksgeiste eine innerliche Verbindung eingegangen sind $[\ldots] .{ }^{4{ }^{47}}$ Indem er die durch Deutschtum und Judentum gebildete Zweierkonstellation von vornherein bricht, versucht er seine Herangehensweise zu legitimieren und zu zeigen, dass die Parallelen, die er zieht, auf anderen Ebenen auch gerechtfertigt sind.

Im Laufe der Abhandlung zieht Cohen zahleiche Parallelen zwischen dem deutschen und dem jüdischen Geistesleben. Die von ihm festgestellten Ähnlichkeiten dienen als Grundlage für ein Fazit über die politische Situation seiner Zeit, die seiner Ansicht nach von Sozialismus und Internationalismus geprägt ist. ${ }^{48}$ Letzteren versteht Cohen als eine Garantie des künftigen Friedens. Dies war im Erscheinungsjahr des Buches ziemlich gewagt, da Kosmopolitismus - damals wie heute - zu den häufigsten antisemitischen Topoi gehörte. Er propagiert aber diese Idee, um seinen Patriotismus gegenüber Deutschland

44. Hermann Cohen, Deutschtum und Judentum. Mit grundlegenden Betrachtungen über Staat und Internationalismus, Gießen, Alfred Töpelmann, 1915, S. 5. Zu der Idee einer Seelenverwandtschaft zwischen Deutschtum und Judentum, vgl. Jörg Marquardt, "Germanophilie im deutschen Judentum im 19. Jahrhundert", Europäische Geschichte Online (EGO), 2011, [http://www.ieg-ego. eu/marquardtj-2011-de], Stand: 10. Februar 2019.

45. Vgl. Rudolf Eisler, Wörterbuch der philosophischen Begriffe [1904], [https://bit.ly/2teOjdO], Stand: 10. Februar 2019.

46. Vgl. Ulrich Sieg, "Der frühe Hermann Cohen und die Völkerpsychologie”, Aschkenas 13 (2), 2003, S. 461-483.

47. Cohen, Deutschtum und Judentum, S. 5

48. Der 34. Absatz der Schrift ist dem "politischen Sozialismus" gewidmet, in dem sich eine weitere Verbindung zwischen Deutschtum und Judentum in der Form der Paarung zwischen „Priestertum" und "Messianismus" zeige. Ibid., S. 32. 
kundzugeben:„Ich glaube nun [...], daß auch dem Juden in Frankreich, in England und in Rußland Pflichten der Pietät gegen Deutschland obliegen; denn es ist das Mutterland seiner Seele, wenn anders seine Religion seine Seele ist." ${ }^{49}$

Deutsche und Juden bilden für ihn eine Schicksalsgemeinschaft, der der Respekt aller Juden in Europa gebührt. Diese Formulierung mag erstaunen - sie ist allerdings der Hoffnung und dem Optimismus bezüglich der Rechte der Juden geschuldet, die der Erste Weltkrieg bis 1916 hervorgerufen hatte. In den ersten Jahren des Konfliktes hofften die deutschen Behörden, dass die Auslandskontakte der deutschen Juden, deren Bedeutung allerdings deutlich überschätzt wurde, zugunsten der deutschen Kriegspropaganda eingesetzt werden könnten. Dieses propagandistische Werk sollte dadurch erleichtert werden, dass es in diesen Jahren für die Juden einfacher wurde, zu hohen Posten in der Armee und in der Verwaltung zu gelangen. ${ }^{50}$ Der Text endet in einer utopischen Überhöhung, die in folgender Aussage kulminiert: „Das Deutschtum muss zum Mittelpunkt eines Staatenbundes werden, der den Frieden der Welt begründen und in ihm die wahrhafte Begründung einer Kulturwelt stiften wird. Der gerechte Krieg ist die Vorbereitung des ewigen Friedens". ${ }^{51}$

Hier ist Kants Einfluss deutlich spürbar. ${ }^{52}$ Dabei sollen die Juden laut Cohen eine besondere Rolle spielen, weil er in ihnen die Nachfahren der biblischen Propheten sehen will. Wenn die Juden der ganzen Welt ihrer Pietät gegenüber Deutschland Ausdruck gewähren würden, dann könnten sie ihre messianische Rolle spielen und die Propheten dieses ewigen Friedens sein:

So sind wir in diesen Zeiten eines epochalen Völkerschicksals auch als Juden stolz darauf, Deutsche zu sein [...]. So fühlen wir uns als deutsche Juden in dem Bewusstsein einer zentralen Kulturkraft, welche die Völker im Sinne der messianischen Menschheit zu verbinden berufen ist. $^{53}$

Es ist ein recht kühnes Argument, den jüdischen Internationalismus ins Zentrum eines eschatologischen Projekts zu stellen, das auf die Erlösung aller Völker durch einen Frieden abzielt, der von dem jüdisch-deutschen Paar garantiert wird. In der Tat handelt es sich um eine Umkehrung eines antisemitischen Motivs, dass das diasporische Leben der Juden als Grund für ihre angebliche Weltmacht ansieht.

Cohens Theorie rief Kritiken aus ganz unterschiedlichen Lagern hervor. Jakob Klatzkin (1882-1948) stellte seine assimilatorische Sichtweise von einem zionistischen Standpunkt aus entschieden in Frage. ${ }^{54}$ Franz Rosenzweig

49. Ibid., S. 42. Dazu vgl. Hartwig Wiedebach, „Das Problem eines einheitlichen Kulturbewußtseins. Zur Person des jüdisch-deutschen Philosophen Hermann Cohen", Aschkenas 10 (2), 2000, S. 417 441.

50. Meyer, Brenner, Deutsch-jüdische Geschichte in der Neuzeit, Bd. 3, S. 362.

51. Cohen, Deutschtum und Judentum, S. 55.

52. Immanuel Kant, Zum ewigen Frieden. Ein philophischer Entwurf (1795), [https://korpora.zim.uniduisburg-essen.de/Kant/aa08/341.html], Stand: 9. Februar 2019.

53. Cohen, Deutschtum und Judentum, S. 45-46.

54. Vgl. Hartwig Wiedebach, „Hermann Cohens Auseinandersetzung mit dem Zionismus. Briefe von Hermann Cohen und Hermann Badt an Martin Buber", Jewish Studies Quarterly 6 (4), 1999, S. $373-$ 388. 
(1886-1929) warf ihm eher seine philosophische Inkonsequenz vor, indem er auf die Gefahren seines philosophischen Essentialismus abhob, ohne jedoch die Legitimität des jüdischen Lebens in Deutschland in Frage zu stellen.

\section{Jakob Klatzkin}

Jakob Klatzkins Rezension, mit dem Titel „Deutschtum und Judentum. Eine Besprechung", erschien im Jahrgang 1917-1918 der von Martin Buber herausgegebenen Zeitschrift Der Jude: Eine Monatschrift, zu einer Zeit also, in der klar war, dass die Hoffnungen, denen sich einige Juden am Anfang des Ersten Weltkriegs hingegeben hatten, in die Irre führten. ${ }^{55}$ In seinen Augen lässt sich Cohens Text nur im Kriegskontext verstehen. Für ihn ist die Neigung, zwischen dem Judentum und allem Möglichen Verbindungen herstellen zu wollen - bei Cohen handelt es sich sowohl um das Deutschtum als auch um das Griechentum und das Christentum -, ein deutliches Zeichen für die "Entwurzelung" des Judentums. ${ }^{56}$ Darüber hinaus wirft er Cohen einen Mangel an Wissenschaftlichkeit vor und zählt seine angeblichen Argumentationsfehler auf. Interessanterweise verwirft er nicht schlichtweg Cohens Werk, sondern zeigt immer wieder, wie diese Schrift anderen Texten des Philosophen widerspricht, in denen dieser viel akzeptablere Ansichten vertritt. Es handelt sich also keineswegs um einen Verriss, obwohl Klatzkin Cohens Thesen insgesamt sehr kritisch gegenübersteht. Vielmehr macht der Text deutlich, dass er die Person Cohen, die für die deutschen Juden der Zeit so wichtig war, nicht als solche verwirft.

Für Klatzkin gebe es tatsächlich wechselseitige Einflüsse zwischen Juden und Deutschen, aber keine „wesentliche“ Verbindung zwischen Deutschtum und Judentum. Überhaupt lehnt er die essentialistische Kategorie des Volksgeists vollkommen ab. Er vertritt die Einsicht, die in der Analyse der jüdischen Diaspora, insbesondere in zionistischen Milieus, eine gewisse Verbreitung erfahren hat, dass die äußeren Einflüsse das Judentum in seinem Kern nicht berühren. Seine Kritik ist immer auch als Profilierung des zionistischen Projekts zu verstehen. Vor diesem Hintergrund versteht sich auch, dass er Cohens Patriotismus als Problem ansieht, das weniger dem Krieg als der diasporischen Situation geschuldet ist, weil sie eine „zwiefache Treue“ verlangt, die im Fall eines Konflikts einer Form von Schizophrenie gleichkommt. ${ }^{57}$

\section{Franz Rosenzweig}

Rosenzweigs Text, der 1915 entstand, aber erst 1937, also acht Jahre nach dem Tod Rosenzweigs erschien, trägt ebenfalls den Titel „Deutschtum und Judentum“. Er geht im Gegensatz zu Klatzkin nicht auf Cohens Argumentation ein, sondern

55. Jakob Klatzkin, „Deutschtum und Judentum. Eine Besprechung“, Der Jude: eine Monatschrift 2 (4), 1917-1918, S. 245-252. Eine extrem harsche Kritik Cohens Text findet man auch im Vorwort seiner französischen Übersetzung: Marc de Launay, "Hermann Cohen ou le romantisme de la raison", Pardes 5, 1987, S. 5-12.

56. Ibid., S. 245.

57. Klatzkin, „'Deutschtum und Judentum. Eine Besprechung“, S. 358-370. 
stellt seine Prämissen radikal in Frage. Im Unterschied zu Klatzkin sieht er in Cohens Deutschtum und Judentum kein Produkt der Umstände, sondern eine Weiterführung des übrigen Werks des Autors. Für ihn sind die Wurzeln seiner Argumentation in der doppelten Quelle von Cohens Werk, dem deutschen Idealismus einerseits und der jüdischen Tradition andererseits, zu finden. Hier zweigt Rosenzweig eine gewisse Nähe zu Klatzkin und seiner Auffassung einer problematischen "zwiefachen Treue“, die die Juden in der Diaspora vor ein ständiges Dilemma stellt. Rosenzweigs Ziel ist es zu zeigen, wie jüdisches Leben in der Diaspora, in dem Fall in Deutschland, gerechtfertigt werden kann: „Der Zwang zur ,Assimilation' ist so alt wie der Galut [Zerstreuung, SG]; dieser hat aber keinen bestimmbaren Anfang in der Zeit". ${ }^{58}$ Er datiert nicht die Anfänge der Diaspora, wie es üblich ist, mit der zweiten Tempelzerstörung im Jahre 70 n.d.Z. Vielmehr bezieht er sich auf den Text der Genesis: „Adam ist erdgeboren, der Jude ist zugewandert ${ }^{\prime \prime}{ }^{59}$ Für ihn gehört die Diaspora zur Wesensbestimmung der Juden. ${ }^{60}$ Dieses Verhältnis der Nicht-Zugehörigkeit zum bewohnten Land begründet die Ahistorizität des jüdischen Volks ${ }^{61}$ :

\begin{abstract}
Was wir empfangen, dürfen wir nicht als Juden empfangen, was wir leisten, sollen wir nicht als Juden leisten. Und gleichwohl sind wir, um der Einheit der Seele willen genötigt, auch unser Judentum zu Empfangenem und Geleistetem in ein wie immer geartetes Verhältnis zu setzen. ${ }^{62}$
\end{abstract}

Diese Diagnose ist derjenigen Klatzkins nicht unähnlich. Rosenzweig distanziert sich allerdings hier bewusst vom Zionismus, indem er sich für das Leben in der Diaspora ausspricht. Die von ihm bevorzugte Position ist die bereits erwähnte "Dissimilation", die die Weiterführung des Lebens in der Diaspora vorsieht, aber eine Affirmation bzw. Neuentdeckung des eigenen Judentums miteinschließt.

In folgendem Zitat findet sich der Kern der Rosenzweigschen Kritik an Cohen:

\begin{abstract}
Wer vom Deutschtum (oder von irgend einem Volkstum) ausgeht, wird dem Judentum das Unrecht tun es als Volk zu wiegen; auf dieser Waage wird es stets zu leicht befunden werden; es ist kein Volk wie andere Völker und ist es, trotz des naiven Vulgärzionismus, auch nie gewesen. Wer hingegen vom Judentum ausgeht - und das tut Cohen, der tut dem Deutschtum die gefährliche Ehre an, es nicht als Volkstum sondern als Religion zu bewerten. ${ }^{63}$
\end{abstract}

Ins Visier nimmt er tatsächlich die eschatologische, ja messianische Dimension von Cohens Text. Er gibt dem Leser zu bedenken, dass die Juden kein Volk wie alle anderen bilden und in dem Sinne nicht mit anderen Völkern zu vergleichen sind. Dies richtet sich natürlich sowohl gegen Cohen als auch gegen die Zionisten,

58. Franz Rosenzweig, „'Deutschtum und Judentum”, in Zweistromland. Kleinere Schriften zu Glauben und Denken, hrsg. v. Annemarie und Reinhold Meyer, Dordrecht, Nijhoff, 1984, S. 169-175, hier S. 169.

59. Ibid.

60. 1 Moses 12: „Der HERR sprach zu Abram: Geh fort aus deinem Land, aus deiner Verwandtschaft und aus deinem Vaterhaus in das Land, das ich dir zeigen werde!"

61. Vgl. Joseph Cohen, Le Spectre juif de Hegel, Paris, Galilée, 2005, S. 69.

62. Rosenzweig, "Deutschtum und Judentum”, S. 170.

63. Ibid., S. 173. 
allerdings steht er in diesem Punkt Klatzkin näher, als man hätte denken können. Cohens Vergleich führe laut Rosenzweig dazu, das Deutschtum zu einer Religion zu machen. Und dies ergebe sich nicht nur aus der Anlage des Vergleichs, den Cohen zwischen zwei ganz unterschiedlich gearteten Formen von Volk aufstellt; sondern der Inhalt des Textes würde in eine ähnliche Richtung weisen, da er die Verehrung aller Juden für Deutschland verlangt.

\section{Perspektiven}

Die Zeit, in der diese zwei Debatten stattinden, bildet einen Übergang zwischen einer wenig hinterfragten Emanzipationsbewegung und der späteren Phase, derjenigen der Weimarer Republik, in der es immer mehr Juden darum geht, eine „selbständige jüdische Sphäre" ${ }^{\prime 64}$ aufzubauen. Mit beiden Debatten, die im vorliegenden Aufsatz präsentiert wurden, wird klar, wie vielfältig die Positionen der deutschen Juden gegenüber Deutschland, der deutschen Kultur und der Möglichkeit eines Lebens in der Diaspora sich gestalteten. Dieses Panorama müsste natürlich erweitert werden, da in den 1910er Jahren viele Texte sich um diese Frage drehten, und es zu den erwähnten Texten andere Stellungnahmen gibt, die auch hätten herangezogen werden können. Zu erwähnen sind dabei zwei Texte von Salman Schocken, die 1915 in der Jüdischen Rundschau erschienen sind. ${ }^{65}$ Der spätere Gründer des Schocken-Verlags vertrat genauso wie Klatzkin einen zionistischen Standpunkt und bezog sich in dem ersten vor allem mit Hermann Cohens Persönlichkeit und mit seiner Stellung innerhalb des deutschen Judentums. In der zweiten fasst er Cohens Buch kritisch zusammen. Vor allem seine allgemeine Bewertung solcher Fragen zu "Deutschtum und Judentum" ist für unser Anliegen wichtig: „In Wirklichkeit ist es auch kein freiwilliger Akt dieser Deutschen jüdischer Konfession, wenn sie über ihr Judensein sprechen", schreibt er. ${ }^{66}$ So gesehen ist diese Frage eine Folge der diasporischen Situation der Juden, die sie dazu zwingt, sich mit ihrer Beziehung zu dem Land und zu der Gesellschaft, die sie aufgenommen hat, auseinanderzusetzen. Darüber hinaus wäre die große Kontroverse um Hermann Cohens Standpunkt zum Zionismus zu erwähnen, an der sich Martin Buber prominent beteiligte. ${ }^{67} \mathrm{Zu}$ der Debatte um Goldsteins

64. Franz Rosenzweig, „Zeit ist's..., in Zweistromland, S. 461-482, hier S. 462. Zu den spezifischen Formen der Erneuerung des jüdischen Lebens in der Weimarer Republik, vgl. Brenner, Jüdische Kultur in der Weimarer Republik.

65. Salman Schocken Jr.,,"Hermann Cohen“, in Jüdische Rundschau vom 5. November 1915, S. 301-302; Ders., "Hermann Cohens Deutschtum und Judentum", in Jüdische Rundschau vom 19. November 1915, S. 377-379.

66. Ibid., S. 377.

67. Hermann Cohen, "Zionismus und Religion. Ein Wort an meine Kommilitonen jüdischen Glaubens", K.C.-Blätter 11, Mai-Juni 1916, S. 643-646; Martin Buber, „Begriffe und Wirklichkeit: Brief an Herrn Geh. Regierungsrat Prof. Dr. Hermann Cohen", Der Jude: eine Monatsschrift 1 (5), 1916-1917, S. 281-289; Hermann Cohen, „Antwort auf das offene Schreiben des Herrn Dr. Martin Buber an Hermann Cohen“, K.C.-Blätter 12, Juli-August 1916, S. 683-688; Martin Buber, „Zion, der Staat und die Menschheit: Bemerkungen zu Hermann Cohens,Antwort'", Der Jude: eine Monatsschrift 1 (7), 1916-1917, S.425-433. 
"Deutsch-jüdischer Parnass" könnte schließlich auch seine 1977 erschienene Autobiographie herangezogen werden, von der man sich einen retrospektiven Blick auf diese Ereignisse erhoffen mag, die aber den zeitlichen Rahmen (18671933) des vorliegenden Bands gesprengt hätte. ${ }^{68}$

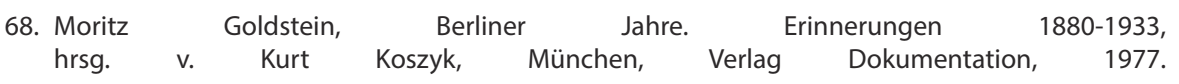

